\title{
Outcome of Switching Anti-VEGF Agents at Fixed Intervals to Treat Eyes With Macular Edema Secondary to Branch Retinal Vein Occlusion
}

\section{Tsuyoshi Mito ( $\square$ mito@kanazawa-med.ac.jp )}

Kanazawa Medical University https://orcid.org/0000-0001-7672-9947

\section{Takeshi Kobayashi}

Ehime University Hospital: Ehime Daigaku Igakubu Fuzoku Byoin

\section{Atsushi Shiraishi}

Ehime University Graduate School of Medicine School of Medicine: Ehime Daigaku Daigakuin Igakukei Kenkyuka Igakubu

\section{Research note}

Keywords: Branch retinal vein occlusion, Macular edema, Anti-VEGF, Injection interval

Posted Date: March 18th, 2021

DOI: https://doi.org/10.21203/rs.3.rs-322286/v1

License: (c) (i) This work is licensed under a Creative Commons Attribution 4.0 International License. Read Full License 


\section{Abstract}

Objective

To determine whether switching intravitreal injections of ranibizumab or aflibercept to the other will affect the interval of subsequent injections in patients with macular edema secondary to branch retinal vein occlusion (BRVO).

Results

The medical charts of 16 eyes of 16 patients were reviewed. After injections of ranibizumab or aflibercept for $>3$ times/year, the type of anti-vascular endothelial growth factor agent was switched. A comparison was made of the average injection interval for the one year before switching and the interval of the first two injections post-switching. In the group that switched from ranibizumab to aflibercept ( $R$ to $A$ group, $n=8$ ), the interval before was $85.4 \pm 6.6$ days which was significantly increased to $125.9 \pm 23.2$ days postswitching $(P=0.0013)$. In the A to R group $(\mathrm{n}=8)$, a significant shortening from $103.9 \pm 11.8$ days before to $85.9 \pm 12.6$ days was observed $(P=0.007)$. In the $\mathrm{R}$ to $A$ group, the central retinal thickness at a recurrence was $522.1 \pm 79.6 \mu \mathrm{m}$ which decreased significantly to $419.3 \pm 64.5 \mu \mathrm{m}$ post-switching $(P=0.005)$. The results suggest that the intervals can be different between ranibizumab and aflibercept in patients with macular edema secondary to BRVO who required repeat injections.

\section{Introduction}

The major cause of the decrease of visual function in eyes with a branch retinal vein occlusion (BRVO) is macular edema. The retinal ischemia caused by the blockage induces an increase in the levels of intraocular vascular endothelial growth factor (VEGF) which then enhances the vascular permeability of the blood vessels. This then results in a leakage and macular edema. ${ }^{[1]}$

Anti-VEGF agents have been shown to be an effective treatment for the BRVO-induced macular edema. ${ }^{[2}$,

3] At present, two anti-VEGF agents, ranibizumab and aflibercept, are widely used to treat patients with a BRVO. The use of aflibercept for central retinal vein occlusion (CRVO) with macular edema requires fewer injections than ranibizumab suggesting that the duration of its effectiveness may differ from that of ranibizumab. ${ }^{[4]}$ However, a direct comparison of the duration of effectiveness has not been determined for these two agents for BRVO. Relevant to our study, two earlier studies found that the interval of injections was prolonged when ranibizumab was switched to aflibercept in patients with macular edema secondary to BRVO. ${ }^{[5,6]}$ However, the prolongation of injection interval after switching agents may be influenced by the time-dependent attenuation of the natural progression of the disease process. ${ }^{[7,8]}$ For this reason, it is important to examine cases in which patients have been switched from aflibercept to ranibizumab.

Thus, the purpose of this study was to compare the interval of anti-VEGF injections after ranibizumab was switched to aflibercept or when aflibercept was switched to ranibizumab in patients with macular 
edema secondary to BRVO. To accomplish this, we reviewed the medical records of patients who had undergone a switching of ranibizumab to aflibercept ( $R$ to $A$ ) or from aflibercept to ranibizumab ( $A$ to $R$ ) on the interval of the injections, and the degree of macular edema.

\section{Methods}

Sixteen eyes of 16 patients with macular edema associated with a BRVO were studied. At least 3 intravitreal injections of an anti-VEGF agent, ranibizumab (Lucentis ${ }^{\circledR}$; Novartis Pharma, Basel, Switzerland) or aflibercept (Eylea $\AA_{\text {; }}$ Bayer Pharma AG, Berlin, Germany) were given for one year, the preswitch period, between July 2015 and December 2018. The patients were then switched to the other type of anti-VEGF agent. The reason for switching agents was because the patient who had an aversion to get repeated same injections accepted our suggestion to change to another type of agent.

The general protocol of our hospital encourages patients to visit their doctor every month at which time optical coherence tomography (OCT; 3D OCT-2000, Topcon) and visual acuity measurements were performed. The OCT images were used to measure the central retinal thickness (CRT), and if the CRT was $\geq 350 \mu \mathrm{m}$, a recurrence of the macular edema was considered to have occurred. Then, an anti-VEGF intravitreal injection was given with the pro re nata (PRN) treatment protocol. Patients who had undergone other ocular treatments such as topical steroids, intraocular surgery, and scatter and grid laser photocoagulation at the pre-switch period were excluded.

Retrospective investigations of the medical records of the patients were performed. A comparison between an average injection interval for the pre-switch period and the interval of the first two injections after switching the anti-VEGF agents was considered the primary endpoint. Comparisons of the CRT before and after switching agents were considered at the time of a recurrence and at the time one month after the injection.

\section{Statistical analyses}

The significance of the differences in the pre-injection demographic data of the R to A and the A to $\mathrm{R}$ groups was determined by Mann-Whitney $U$ tests or Fisher's exact tests. The significance of the changes in the injection intervals and CRT before and after switching agents was made using paired $t$-tests. A $P$ value $<0.05$ was taken to be statistically significant.

\section{Results}

The patients were divided into two groups: the R to A group ( 4 men and 4 women) and the A to R group (5 men and 3 women). The demographics of the patients in the two groups are summarized in Table 1. There were no significant differences in the mean age, male-female ratio, best-corrected visual acuity, CRT, estimated duration from onset, frequency of anti-VEGF injections in the pre-switch period, and previous photocoagulations between the two groups.

\section{Table 1. Demographics of patients}




\begin{tabular}{|l|c|c|c}
\hline & ranibizumab to aflibercept $(n=8)$ & aflibercept to ranibizumab $(n=8)$ & $p$ value \\
\hline Age in years & $69.9 \pm 7.2$ & $68.8 \pm 7.6$ & $0.764^{\mathrm{a}}$ \\
\hline Sex, Male/Female & $4 / 4$ & $5 / 3$ & $0.61^{\mathrm{b}}$ \\
\hline BCVA at baseline (log-MAR) & $0.25 \pm 0.21$ & $0.34 \pm 0.28$ & $0.751^{\mathrm{a}}$ \\
\hline CRT at baseline & $647.1 \pm 98.8$ & $617.0 \pm 74.5$ & $0.713^{\mathrm{a}}$ \\
\hline Duration of symptoms before switch (months) & $33.0 \pm 9.2$ & $26.8 \pm 7.8$ & $0.346^{\mathrm{a}}$ \\
\hline Number of injections before switch (times /year) & $3.50 \pm 0.75$ & $3.25 \pm 0.46$ & $0.438^{\mathrm{a}}$ \\
\hline Previous photocoagulation for capillary non-perfusion & 5 & 6 & $0.59^{\mathrm{b}}$ \\
\hline
\end{tabular}

Values are the means \pm standard deviations.

n, number of eyes; BCVA, best-corrected visual acuity; log-MAR, logarithm of the minimum angle of resolution; CRT, central retinal thickness.

${ }^{a}$ Mann-Whitney U test, ${ }^{b}$ Fisher's exact test.

In the $\mathrm{R}$ to A group. the mean injection interval before switching was $85.4 \pm 6.6$ days which was significantly increased to $125.9 \pm 23.2$ days after the switch $(P=0.0013$; Fig. $1 \mathrm{a})$. In the A to $\mathrm{R}$ group, there was a significant shortening of injection interval from $103.9 \pm 11.8$ days before to $85.9 \pm 12.6$ days after switching agents ( $P=0.007$; Fig. $1 \mathrm{~b})$. In the R to A group, the percentage increase in the injection interval before and after switching agents was $\pm 10 \%$ in 1 eye (12.5\%), $10-30 \%$ in 1 eye (12.5\%), and $\geq 30 \%$ in 6 eyes (75\%). In the A to R group, the percentage shortening of the intervals before and after switching agents was $\pm 10 \%$ in 2 eyes (25\%), $10-30 \%$ in 5 eyes (62.5\%), and $\geq 30 \%$ shortening in 1 eye (12.5\%; Fig. 1c).

The mean CRTs at one month after the anti-VEGF injections was $228.7 \pm 31.8 \mu \mathrm{m}$ before and $230.4 \pm 45.2$ $\mu \mathrm{m}$ after the switch in the $\mathrm{R}$ to $\mathrm{A}$ group $(P>0.05$; Fig. 2a) and $263.4 \pm 28.0 \mu \mathrm{m}$ before and $274.5 \pm 30.4 \mu \mathrm{m}$ after the switch in the $A$ to $\mathrm{R}$ group ( $P>0.05$; Fig. $2 \mathrm{c})$. The mean CRTs at a recurrence in the $\mathrm{R}$ to $\mathrm{A}$ group, was $522.1 \pm 79.6 \mu \mathrm{m}$ before and $419.3 \pm 64.5 \mu \mathrm{m}$ after the switch, and there was a significant difference in the degree of macular edema $(P=0.005$; Fig. $2 \mathrm{~b})$. In contrast, the CRTs was $433.8 \pm 79.6 \mu \mathrm{m}$ and 472.5 $\pm 75.3 \mu \mathrm{m}$ at a recurrence in the A to R group, and these findings showed that the differences in the degree of macular edema was not significantly changed ( $P>0.05$; Fig. $2 \mathrm{~d})$.

\section{Discussion}

We observed a prolongation of the injection interval after switching to aflibercept and shortening of injection interval after switching to ranibizumab. These findings suggest that the prolongation and shortening of the injection interval were related to a difference in the duration of effectiveness of the two agents.

In the $\mathrm{R}$ to A group, average injection interval before the switch was 85 days, and it increased significantly to 126 days after the switch. The increase in the injection intervals was 41 days which is 1.48 times longer than the average injection interval during the pre-switch period. In a prospective study, Casselholm et al ${ }^{[4]}$ compared the injection intervals for ranibizumab and aflibercept in eyes with a CRVO performed 
with the treat-and-extend protocol. They reported that the average injection interval for ranibizumab was 6.6 week and that for aflibercept was 10.0 weeks. These findings indicated that the effects of aflibercept lasted 1.5X longer than ranibizumab. Thus, their findings are comparable to our percentage prolongation of the injection interval in the $\mathrm{R}$ to $\mathrm{A}$ group.

There have been many studies on the effectiveness of switching from ranibizumab to aflibercept in patients with macular edema secondary to a BRVO. The patients studied can be classified into two types; one type is the patient who was not responding to ranibizumab and were then switched to aflibercept, $[5,9,10]$ and the other type was the patient who had a difference in the duration of the effectiveness between two agents as indicated by the lengthening of the interval after switching to aflibercept. ${ }^{[6]}$ The difficulty in comparing these results is that there is a spontaneous healing in more than $30 \%$ of cases if macular edema secondary to BRVO develops within one year after the onset of BRVO. ${ }^{[11]}$ The RELATE study examined patients who had received ranibizumab treatment with a loading phase of 6 PRN injections for 3 years, and they found that the number of injections required for successful treatment decreased with time; 9.7 times in the first year, 6.9 times in the second year, and 4.0 times in the third year. [12] Thus, we cannot eliminate the possibility that the prolongation of injection interval after switching to aflibercept was related to a natural attenuation of the disease progression. Therefore, after switching to ranibizumab, a prolongation of injection interval could also be expected. However, to our knowledge, there have been no studies performed to evaluate the change in the injection interval after switching therapy from aflibercept to ranibizumab in patients with BRVO. In our cases, in the A to R group, average injection interval before the switch was 104 days and after the switch to ranibizumab was 86 days. Therefore, the difference between the two injection intervals is 18 days, that is, 1.21 times shorter than the average injection interval after switching to ranibizumab. The results showed no prolongation of injection interval due to a time-dependent attenuation of the natural progression of the disease process.

The reason for the longer effectiveness of aflibercept is because it has different biochemical characteristics from ranibizumab. For example, aflibercept can bind not only to VEGF-A but also to other VEGF family members, such as VEGF-B and placental growth factor (PIGF). In addition, aflibercept has a strong affinity for VEGF-A. ${ }^{[13]}$ The intraocular PIGF levels are elevated in patients with macular edema secondary to BRVO suggesting that it may be involved in the recurrence of macular edema. ${ }^{[14]}$ In animal experiments, Stewart et al. reported that aflibercept had longer binding activity than ranibizumab. ${ }^{[15]}$ In addition, Niwa et al. compared the intraocular kinetics of ranibizumab and aflibercept after intravitreal injections in monkeys. ${ }^{[16]}$ They reported that the half-life of both agents in the aqueous after intravitreal injections was not significantly different, but aflibercept suppressed the VEGF level for a longer time. From these results, we can conclude that aflibercept has a longer period of effectiveness. This was true even after the agents decreased below the detection level which suggested that aflibercept has a better potential of preventing the recurrence of macular edema. Saishin et al. examined the effects of intravitreal injections of ranibizumab and aflibercept bimonthly on CRVO patients, and they reported that the CRT measured at the time of recurrence was thicker in the ranibizumab than the aflibercept group. ${ }^{[17]}$ 
Their findings are consistent with the results of our study in showing the difference in the CRTs at the time of recurrence in the $\mathrm{R}$ to $\mathrm{A}$ group.

In conclusion, the injection interval can be different between ranibizumab and aflibercept in patients with macular edema secondary to BRVO. Because the difference of injection interval affects patient's medical cost, economic, or psychological burden, it is necessary to focus on the selection of agents for BRVO treatment as well as relieving the effects of macular edema by the anti-VEGF agents.

\section{Limitations}

Our study was limited due to its retrospective nature and small sample size. Another limitation is the different assessment period among pre-switching and after switching agents. On one hand, a one-year assessment period was set before switching agents, but the assessment period after switching agents was not the same as the one before switching agents. This is because patients have a tendency to ask physicians to switch back to the previous one when a shortening of the injection interval occurs due to switching agents. This indicates that the prolongation or shortening of the injection interval becomes a sufficient reason for patients to select the agent.

\section{Abbreviations}

BRVO: branch retinal vein occlusion; CRT: central retinal thickness; CRVO: central retinal vein occlusion; OCT: optical coherence tomography; PIGF: Placental growth factor; PRN: pro re nata; VEGF: vascular endothelial growth factor.

\section{Declarations}

\section{Authors' contributions}

TM collected the data, drafted the manuscript, made figures, and sought data analysis consultations. TK and AS conceptualized the study design, modified manuscript and data analyses. All authors read and approved the final manuscript.

\section{Acknowledgments}

We thank Professor Emeritus Duco Hamasaki of the Bascom Palmer Eye Institute for his critical discussion and final manuscript revision.

\section{Data Availability}

The data used to support the findings of this study are available from the corresponding author upon request.

\section{Ethical Approval}


This study complied with the tenets of the Declaration of Helsinki. This study was approved by Institutional Review Board/Ethics Committee of University of Ehime Graduate School of Medicine Hospital (approval No.: 1903012). Written informed consent was obtained from the patient to publish and report individual patient data.

\section{Funding}

No funding was received by any of the authors for writing this manuscript.

\section{Conflicts of Interest}

The authors declare no conflicts of interest relevant to the present study.

\section{Consent for publication}

Not applicable.

\section{References}

1. Wong TY, Scott IU. Clinical practice. Retinal-vein occlusion. N Engl J Med. 2010 Nov;363(22):2135-44.

2. Brown DM, Campochiaro PA, Bhisitkul RB, Ho AC, Gray S, Saroj N, et al. Sustained benefits from ranibizumab for macular edema following branch retinal vein occlusion: 12-month outcomes of a phase $\otimes$ Ophthalmology. 2011 Aug;118(8):1594-602.

3. Clark WL, Boyer DS, Heier JS, Brown DM, Haller JA, Vitti R, et al. Intravitreal Aflibercept for Macular Edema Following Branch Retinal Vein Occlusion: 52-Week Results of the VIBRANT Study. Ophthalmology. 2016 Feb;123(2):330-6.

4. Casselholm de Salles M, Amren U, Kvanta A, Epstein DL. INJECTION FREQUENCY OF AFLIBERCEPT VERSUS RANIBIZUMAB IN A TREAT-AND-EXTEND REGIMEN FOR CENTRAL RETINAL VEIN OCCLUSION: A Randomized Clinical Trial. Retina. 2019 Jul;39(7):1370-6.

5. Wirth MA, Becker MD, Graf N, Michels S. Aflibercept in branch retinal vein occlusion as second line therapy: clinical outcome 12 months after changing treatment from bevacizumab/ranibizumab-a pilot study. Int J Retina Vitreous. 2016 Aug;23;2:20.

6. Tagami M, Sai R, Fukuda M, Azumi A. Prolongation of injection interval after switching therapy from ranibizumab to aflibercept in Japanese patients with macular edema secondary to branch retinal vein occlusion. Clin Ophthalmol. 2017 Feb;22;11:403-8.

7. Hayreh SS, Zimmerman MB. Branch retinal vein occlusion: natural history of visual outcome. JAMA Ophthalmol. 2014 Jan;132(1):13-22.

8. Finkelstein D. Ischemic macular edema. Recognition and favorable natural history in branch vein occlusion. Arch Ophthalmol. 1992 Oct;110(10):1427-34.

9. Konidaris VE, Tsaousis KT, Anzidei R, de la Mata G, Brent AJ. Real-World Results of Switching Treatment from Ranibizumab to Aflibercept in Macular Oedema Secondary to Branch Retinal Vein 
Occlusion. Ophthalmol Ther. 2018 Dec;7(2):387-95.

10. Sakanishi Y, Usui-Ouchi A, Tamaki K, Mashimo K, Ito R, Ebihara N. Short-term outcomes in patients with branch retinal vein occlusion who received intravitreal aflibercept with or without intravitreal ranibizumab. Clin Ophthalmol. 2017 May;3;11:829-34.

11. The Branch Vein Occlusion Study Group. Argon laser photocoagulation for macular edema in branch vein occlusion. Am J Ophthalmol. 1984 Sep;15;98(3):271-82.

12. Campochiaro PA, Hafiz G, Mir TA, Scott AW, Solomon S, Zimmer-Galler I, et al. Scatter Photocoagulation Does Not Reduce Macular Edema or Treatment Burden in Patients with Retinal Vein Occlusion: The RELATE Trial. Ophthalmology. 2015 Jul;122(7):1426-37.

13. Papadopoulos N, Martin J, Ruan Q, Rafique A, Rosconi MP, Shi E, et al. Binding ang neutralization of vascular endothelial growth factor (VEGF) and related ligands by VEGF Trap, ranibizumab and bevacizumab. Angiogenesis. 2012 Jun;15(2):171-85.

14. Noma H, Mimura T, Yasuda K, Shimura M. Role of soluble vascular endothelial growth factor receptors- 1 and -2 , their ligands, and other factors in branch retinal vein occlusion with macular edema. Invest Ophthalmol Vis Sci. 2014 Jun;3;55(6):3878-85.

15. Stewart MW, Rosenfeld PJ. Predicted biological activity of intravitreal VEGF Trap. Br J Ophthalmol. 2008 May;92(5):667-8.

16. Niwa Y, Kakinoki M, Sawada T, Wang X, Ohji M. Ranibizumab and Aflibercept: Intraocular Pharmacokinetics and Their Effects on Aqueous VEGF Level in Vitrectomized and Nonvitrectomized Macaque Eyes. Invest Ophthalmol Vis Sci. 2015 Oct;56(11):6501-5.

17. Saishin Y, Ito Y, Fujikawa M, Sawada T, Ohji M. Comparison between ranibizumab and aflibercept for macular edema associated with central retinal vein occlusion. Jpn J Ophthalmol. 2017 Jan;61(1):6773.

\section{Figures}




\section{Figure 1}

a

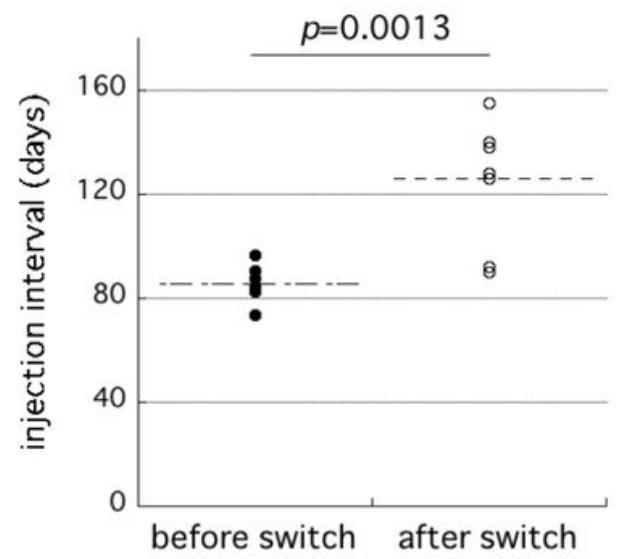

b

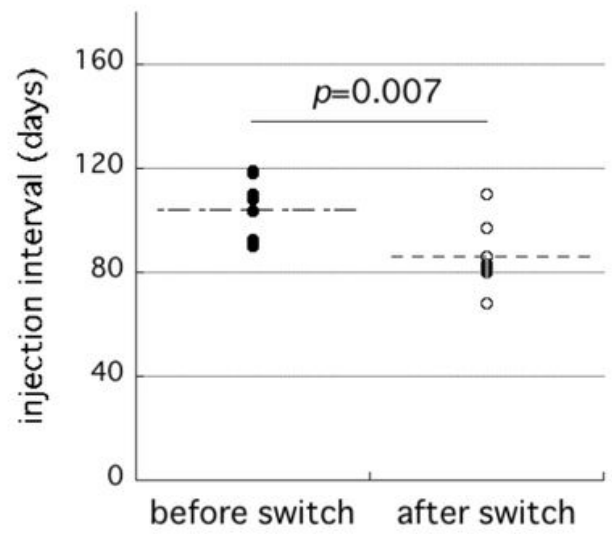

C

(n)

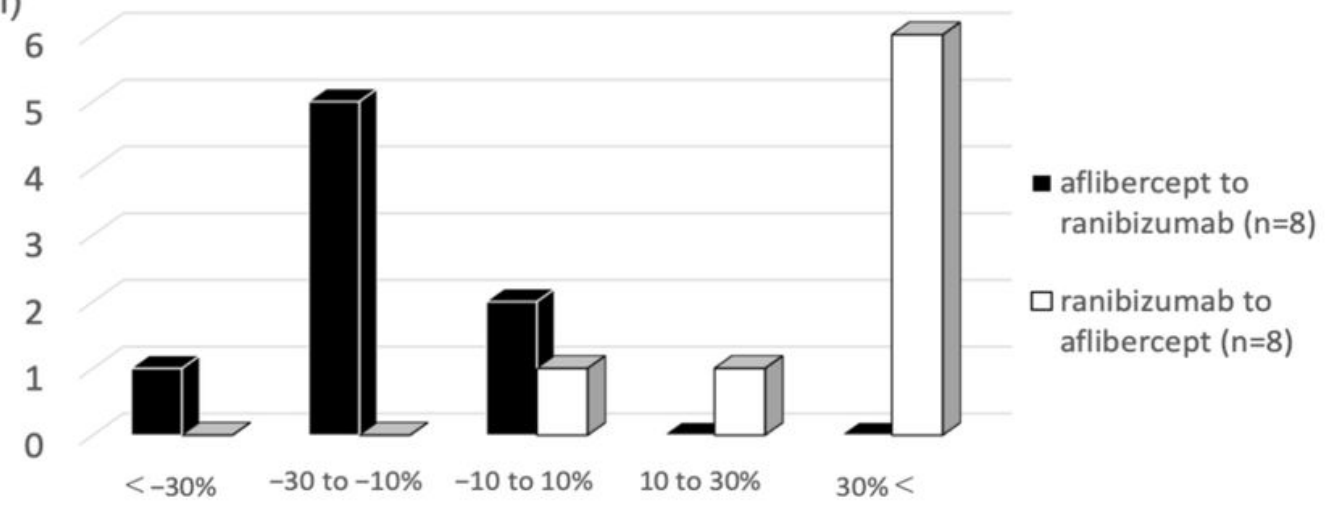

Figure 1

Injection intervals before and after switching anti-vascular endothelial growth factors (anti-VEGFs). a: Group that switched from ranibizumab to aflibercept ( $R$ to $A$ ). P-value, paired $t$ test. b: Group that switched aflibercept to ranibizumab (A to R). P-value, paired $t$ test. c: Percentage change of injection interval before and after switching anti-VEGF agents. 
Figure 2

a

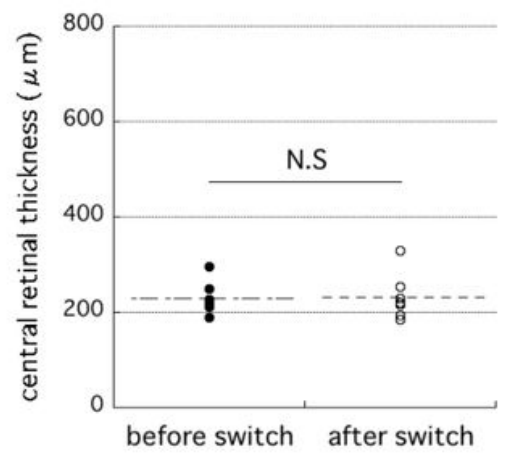

C

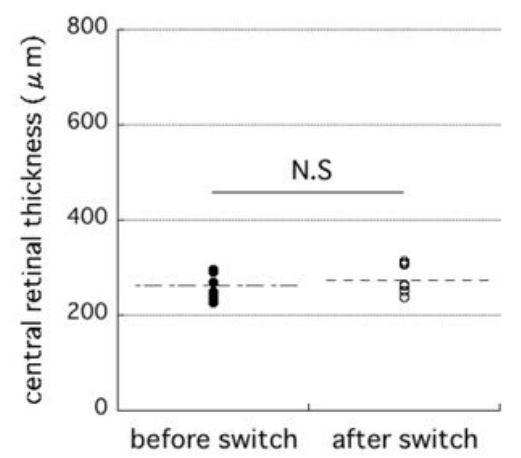

b

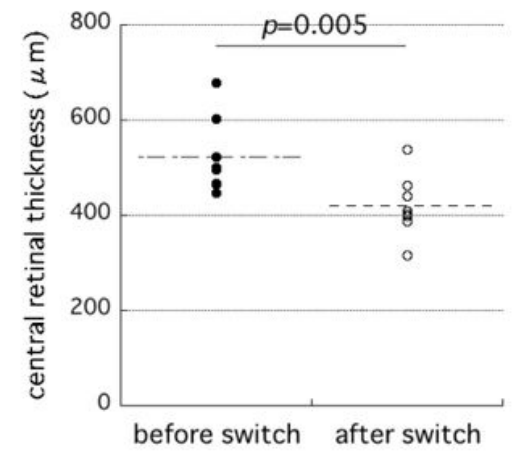

d

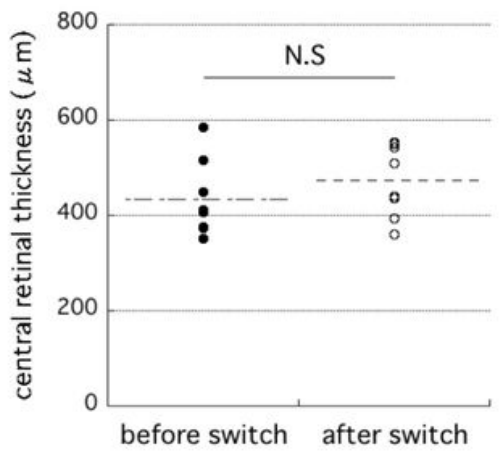

\section{Figure 2}

Central retinal thickness (CRT) before and after switching anti-VEGF agents. a: In the R to A group, the CRT before and after switching agents at one month after the anti-VEGF injection. b: In the R to A group, the CRT before and after switching agents at a recurrence. $\mathrm{C}$ : In the A to R group, the CRT before and after switching agents at one month after the anti-VEGF injection. $\mathrm{d}$ : In the A to R group, the CRT before and after switching agents at a recurrence. P-value, paired $t$ test. 